\title{
Unit 2.1: Electro-magnetic wave fundamentals and the equation of radiative transfer
}

\author{
Javier Alcolea* \\ Observatorio Astronómico Nacional (Spain) \\ E-mail: j.alcolea@oan.es
}

\begin{abstract}
As a brief introduction, in this chapter I will review the importance of the electro-magnetic waves as astronomical information carriers, and the basic properties of them. I will also briefly revise the basic concepts of the equation of the radiative transfer, from the definition and properties of an ideal black-body, to the equation of transfer in the more complex case of the presence of both true absorption and scattering.
\end{abstract}

2nd MCCT-SKADS Training School. Radio Astronomy: fundamentals and the new instruments August 26- September 4, 2008

Sigüenza, Spain

${ }^{*}$ Speaker. 


\section{Introduction: sources of information in Astronomy}

There are several ways in which we obtain information from astrophysical objects and processes. We can directly send (manned or unmanned) probes to test our neighborhood, but using this method we have only explored just part of our own Solar System. We can also probe the astronomical particles that reach the Earth, such as meteorites and micro-meteorites originally from comets, asteroids and even nearby planets (Mars). We can also measure the energy of the atomic and subatomic particles that reach us from outside, such as the cosmic rays (electrons and light nuclei) from the Sun, our Galaxy and other Galaxies, and also the neutrinos from our Sun and from galactic and extragalatic supernova explosions. We can also test the effects of long range forces, but since the matter is normally neutral at large scales, we are just left with the Gravitation. Here we can directly measure the effects of the Sun, Moon, and giant planets. We can also measure the Gravitational field in other bodies by sending probes, which again so far is limited to the Solar System. Our last hope is detecting waves that can propagate in the vacuum. Gravitational waves are predicted but remain yet to be detected, so we are left with the electro-magnetic waves (EMWs) which in fact constitute, by large, the most important carrier of astrophysical information. This is way astronomical observations are mainly (but not only) carried out by collecting EMWs of various types reaching us from above the atmosphere.

\section{Electro-magnetic waves: classical approach}

Let us write J.C. Maxwell's equations of the Electric $(\vec{E})$ and Magnetic $(\vec{H})$ fields:

$$
\nabla \cdot \varepsilon \vec{E}=4 \pi \rho ; \quad \nabla \cdot \mu \vec{H}=0 ; \quad \nabla \times \vec{E}=-\frac{1}{c} \frac{\partial \mu \vec{H}}{\partial t} ; \quad \nabla \times \vec{H}=\frac{4 \pi}{c} \vec{J}+\frac{1}{c} \frac{\partial \varepsilon \vec{E}}{\partial t}
$$

In absence of electrical charged (static or in movement) particles (e.g. in vacuum) these equation can be rewritten as follows:

$$
\nabla \cdot \vec{E}=0 ; \quad \nabla \cdot \vec{H}=0 ; \quad \nabla \times \vec{E}=-\frac{1}{c} \frac{\partial \vec{H}}{\partial t} ; \quad \nabla \times \vec{H}=+\frac{1}{c} \frac{\partial \vec{E}}{\partial t}
$$

From these equations, we also have the following relations:

$$
\nabla^{2} \vec{E}=\frac{1}{c^{2}} \frac{\partial^{2} \vec{E}}{\partial t^{2}} ; \quad \nabla^{2} \vec{H}=\frac{1}{c^{2}} \frac{\partial^{2} \vec{H}}{\partial t^{2}}
$$

These are precisely the classical equations of waves, whose solution is a series of sinusoidal (wave) functions for both $\vec{E}$ and $\vec{H}$. However these two fields are not independent. Both waves should have the same period and phase. In addition both fields must be orthogonal to each other, and to the direction of propagation of the field. The velocity of propagation only depends on fundamental physical constants and therefore must be constant too: this is the speed of light in the vacuum $c=299792458 \mathrm{~m} \mathrm{~s}^{-1}$, which nowadays is a fundamental unit in Physics (the length is now defined in terms of $c$ and the fundamental unit of time).

Since they travel, EMWs transport the energy $U$ due to the $\vec{E}$ y $\vec{H}$ fields at $c$ speed, which results in a flux of energy $\vec{S}$. These quantities are just given by the expressions: 


$$
U=\frac{1}{8 \pi}\left(E^{2}+H^{2}\right) ; \quad \vec{S}=\frac{c}{4 \pi} \vec{E} \times \vec{H}
$$

Classically, EMWs are due to the accelerated movement, ordered or random, of electrically charged particles. Since real bodies mainly consist in charged particles (electrons and nucleons), all these bodies must emit EMWs up to some extent (unless they are at the minimum energy level and at zero absolute temperature).

\section{The electro-magnetic spectrum}

The wave equation of the EMWs has as possible solution any linear combination of pure monochromatic waves, i.e. with a fixed frequency or period of oscillation. Each of these components can be characterized by:

- the frequency $v$, equal to number of oscillations per second of the EM field. It is measured in Hertzs (Hz): $1 \mathrm{~Hz}=1 \mathrm{~s}^{-1}$

- the wavelength $\lambda$, or the distance separation between two adjacent maxima (or minima) of the value of the EM field. It is measured in units of distance (m, cm, mm, $\mu \mathrm{m}$, $\AA$, etc.)

Obviously, the following relation must also hold:

$$
\lambda v=c
$$

We can also characterize an EMW by its wave number, $k$, which is the number of oscillations of the fields comprised in one meter. This number is just the inverse of the wavelength and it is measured in units of the inverse of distance (e.g. $\mathrm{cm}^{-1}$ ).

Traditionally, different names were assigned to the different types of EMWs (visible, X-rays), depending on the range of frequencies, since this value is intimately related with how light interacts with matter. We call EM spectrum to all kinds of EMWs, i.e. the full frequency range, from decametric radio waves (lowest frequency) up to the $\gamma$-rays (highest frequency). Visible light, $\mathrm{X}$-rays, radio emission are just EMWs of certain characteristics.

\section{The quantization of the EM field}

Quantum Field Theory states that the energy of the EM field can not take any arbitrary value, but an integer multiple of a basic quantity. This is valid for any monochromatic component. For each frequency, this basic, minimum quantum of energy $E_{\min }$ is given by the relation:

$$
E_{\min }=h v
$$

where $h$ is the Plank's constant, whose value is $\mathrm{h}=6.62606876$ (52) $10^{-34} \mathrm{~J} \mathrm{~s}$

In the EM field, these basic units of energy are known as photons. This is way sometimes it is said that the light (or EMWs in general) has a dual wave-particle nature: as waves, EMWs suffer 
process of interference, refraction and scattering, as particles they are quantized and must consist in an integer number of photons.

Furthermore, the quantum theory of the EM field states that, not only EMWs but all electromagnetic interactions are due to photons. The electric and magnetic forces between static particles and currents are due to the interchange of photons between these particles (interchange of EMWs). If there are currents and/or charged particles, photons must exist, since they transfer the forces of the fields. On the contrary, we can have photons without having any static of moving charged particles, i.e. we can have EMWs traveling in the empty space. Some basic properties of the photons are:

- Energy:

$$
\begin{array}{r}
E=h v \\
P=h v / c \\
m=h v / c^{2} \\
\pm 1 \\
0
\end{array}
$$

- Lineal momentum (photons are capable of pushing other particles):

- Mass (they suffer the gravitational attraction):

- Spin (intrinsic angular momentum):

- Charge (of any kind):

Since for any frequency we have a different energy for the photons, we can also characterize monochromatic EMWs by that basic unit of energy. This energy can be given $\mathrm{eV}, \mathrm{keV} \mathrm{o} \mathrm{MeV}$, for very energetic photons (UV, X-rays, and $\gamma$-rays), or in units of absolute temperature (K), taking advance of the relationship, $k T=E=h v$, where $k$ is Boltzmann's constant, whose value is $k=$ $1.3806503(24) 10^{-23} \mathrm{~J} \mathrm{~K}^{-1}$. To convert these scales into frequencies in $\mathrm{Hz}$ we can use the following equations:

$$
2.417989491(95) 10^{14} \mathrm{~Hz} / \mathrm{eV} \quad ; \quad 2.0836644(36) 10^{10} \mathrm{~Hz} / \mathrm{K}
$$

Depending on the wavelength/frequency/energy of the radiation, EMWs are known with different names, are produced by different physical processes, and must be detected with very different devices (receivers/detectors). In Astronomy, these detectors (or telescopes as we call them), must be placed in very different locations, at high altitude, balloons, aircrafts, or even rockets and satellites, depending on the transparency of our atmosphere at the band of interest.

\section{The spectrum of physical systems}

In general, physical systems (solid bodies, liquids, gases, plasmas, etc.) emit and absorb (and diffract and scatter) EMWs. We define the "spectrum of a system" as the power radiated by the system as a function of the frequency/wavelength. These plots/functions are also called the "spectral energy distribution" (SED) of the system.

Quantic systems (i.e. those systems which have discrete levels of energy), such as gases of molecules, radicals, atoms, and nuclei, emit (and absorb) photons just at some discrete frequencies. This is because in each change in the energy state of the system, just a single photon is emitted or absorbed, and therefore the energy of this photon, corresponding to a precise value of the frequency, must coincide with the change in energy of the system. 
On the contrary, systems with continuous levels of energy, such as quantized macroscopic systems (in which the energy levels are so much tightly packed that there is no discontinuity between them), or a gas of free electrons, can emit photons (in principle) of any frequency.

If the emission of a system is mainly due to the thermal random motion of the particles, we say that the spectrum is of thermal origin. If this is not the case, we say that the spectrum is of non-thermal origin. Roughly speaking, non-thermal continuous emission are associated with relativistic and/or very energetic processes. Not-thermal discrete emissions are due to thermal quantized systems not in thermodynamic equilibrium, such a masers.

In general, emissions of thermal origin show the following properties:

- Depending on the temperature of the system, there is a characteristic frequency above which photons are seldom emitted.

- For similar systems, the hotter the body the more energy it emits at all frequencies. This does not hold for quantized systems. However if we fix the total luminosity of the system, cooler media emit more radiation at lower frequencies than hotter systems.

- The hotter the system the higher the maximum frequency at which photons can be emitted. Only very hot systems can emit photons of very high energy. But non-thermal emissions can also give rise to very hard (energetic) photons.

\section{The Black-Body radiation spectrum}

We define a Black-Body as the perfect emitter, i.e., the one that in thermodynamic equilibrium emits most EM radiation at any wavelength. Of course, in the real world perfect black-bodies do not exist, the concept is rather a mathematical idealization, but a god approximation could be a hole in a cavity covered by mirrors, or a very opaque system. The spectral energy distribution spectrum (SED) of a Black-Body at a temperature $T$ is given by the formulae:

$$
B_{\lambda}(T)=\frac{2 h c^{2}}{\lambda^{5}} \frac{1}{e^{\frac{h c}{\lambda k T}}-1} \equiv B_{v}(T)=\frac{2 h v^{3}}{c^{2}} \frac{1}{e^{\frac{h v}{k T}}-1}
$$

where $B_{v}$ y $B_{\lambda}$ represent the energy radiated per unit of time, per unit of surface (of the emitting body), and per unit of solid angle (unit of directions), and per unit of frequency $\left(B_{v}\right)$ or unit of wavelength $\left(B_{\lambda}\right)$, respectively. The factor 2 is due to the 2 possible independent polarizations of the light (spin $= \pm 1$, horizontal and vertical, or right- and left-circular). $h$ is the Plank's constant and $\mathrm{y} c$ is the speed of light in vacuum (see Fig. 1). These expressions are known as the Planck's law of the Black-Body emission.

For the case of black-bodies, we can derive the temperature of the system from the shape of the spectrum of emission, finding the point at which it attains the maximum emission:

$$
\begin{gathered}
\max \left(B_{\lambda}\right) \equiv \lambda=0.289 \mathrm{~cm} \mathrm{~K}^{-1} T \quad\left(v=103 \mathrm{GHzK}^{-1} T\right) \\
\max \left(B_{v}\right) \equiv v=58.8 \mathrm{GHzK}^{-1} T \quad\left(\lambda=0.51 \mathrm{~cm} \mathrm{~K}^{-1} T\right)
\end{gathered}
$$




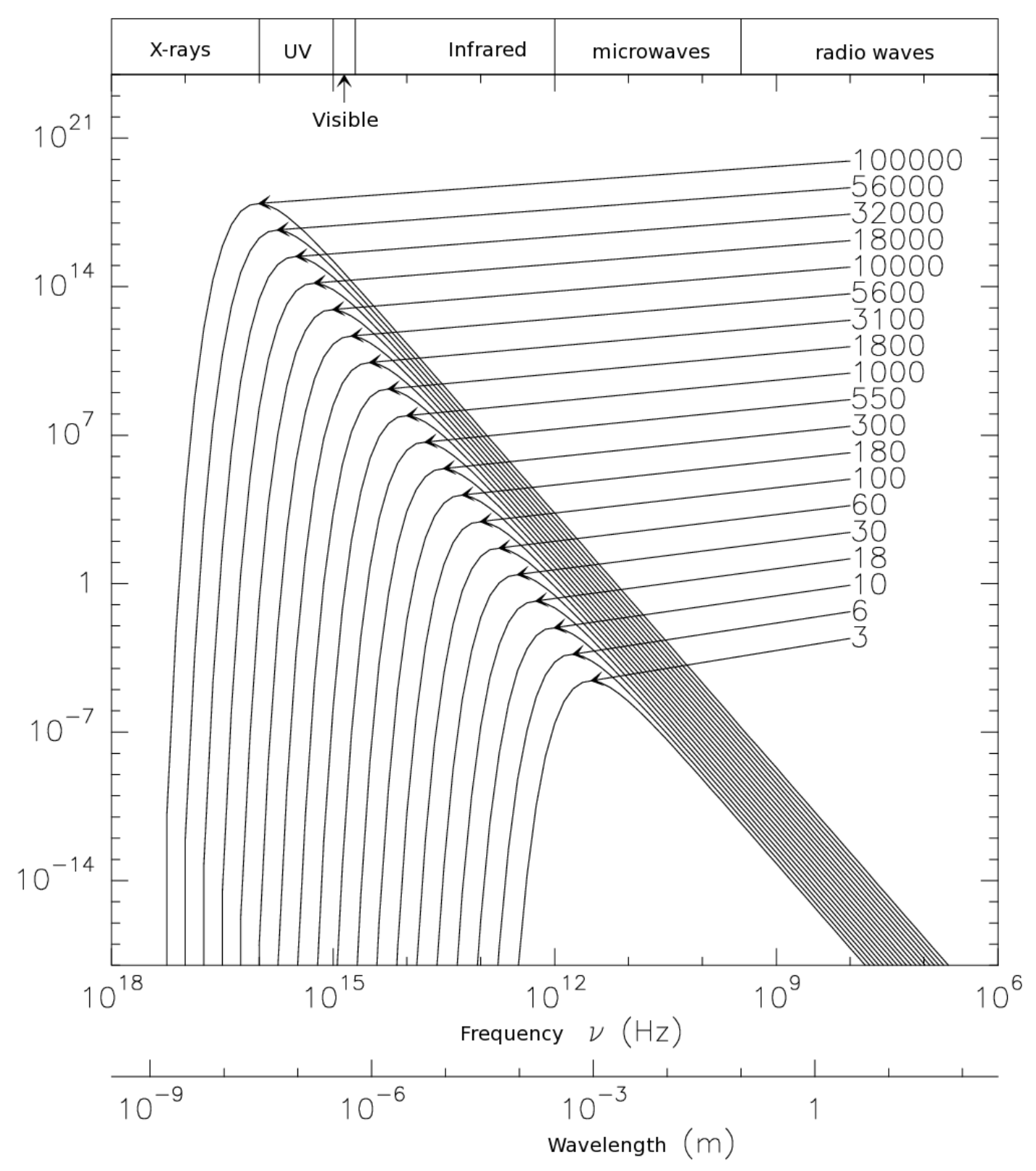

Figure 1: SEDs of several black-bodies of equal size but different temperature. Here we have plot SEDs for bodies at absolute temperatures (in Kelvin) of 3 (cosmic background), 6, 10, 18, 30, 60, 100, 180, 300 (the Earth), 550, 1000, 1800 (brown dwarf), 3100 (M-type star), 5600 (the Sun), 10000 (A-type star), 18000 (B-type star), 32000,56000 (O-type star) and 100000 (blue dwarf). Intensity is given in relative units. 
A hotter black-body emits more energy in all the EM spectrum that a cooler black-body, assuming that both are of the same size. For each temperature there is a maximum frequency (minimum wavelength) above which (under which) the black-body practically emits nothing (Wien region). The higher the temperature of the black-body, the higher the frequency (the lower the wavelength) at which the black-body can emit photons.

Integrating the power emitted at all frequencies/wavelengths, and multiplying by the total area of the surface of body and by the $4 \pi$ stereo radians of the full range of possible directions, we obtain that the total luminosity $L$ of a spherical black-body of radius $R$ and temperature $T$ is:

$$
L=4 \pi \sigma R^{2} T^{4}
$$

where $\sigma$ is the Stefan-Boltzman's constant, whose value is $5.6710^{-8} \mathrm{~W} \mathrm{~m}^{-2} \mathrm{~K}^{-4}$. Using this relationship, we can derive sizes of astrophysical black-bodies knowing their luminosity and temperature (that we can derive from the peak of the emission spectrum), under the black-body approximation.

\section{The equation of the radiative transfer}

In general electro-magnetic waves (EMWs) do not propagate just in vacuum, but they travel across physical systems, more or less diffuse, or more or less transparent. When an EMW travels in one direction across a medium that is not fully transparent, it suffers a decrease in flux, because of the photons absorbed by the medium. At the same time, it also suffers an increase of flux, because of the photons emitted by the medium in the same direction. (For simplicity, here we are neglecting, for the time being, the effects of the possible scattering of the emission). These processes are ruled by the so called equation of radiative transfer.

Let us assume that a ray of light with intensity $I_{v}$ (at $v$ frequency) arrives at a volume of area $d \sigma$, and length $d s$ in the direction of travel of the ray. the incident intensity is $I_{v}\left(s_{0}\right)$, whereas the emergent intensity will be $I_{v}\left(s_{0}+d s\right)$. (Remember that we are assuming no scattering.) The differential equation controlling the propagation of the ray is:

$$
\frac{d I_{v}}{d s}=-k_{v} I_{v}+\varepsilon_{v}
$$

where $k_{v}$ is called the absorption coefficient per unit of length, and $\varepsilon_{v}$ is the emissivity of the medium (see Fig. 2).

For a black-body in thermodynamic equilibrium, the intensity of the radiation can not change, since it is given by Planck's law. Therefore we can conclude that:

$$
\begin{gathered}
\frac{d I_{v}}{d s}=0 \quad \Longrightarrow \quad k_{v} I_{v}=\varepsilon_{v} \\
I_{v}=B_{v}(T) \quad \Longrightarrow \quad k_{v} B_{v}(T)=\varepsilon_{v}
\end{gathered}
$$

This last equation is known as Kirchoff's law.

Since $k_{v}$ and $\varepsilon_{v}$ only depend on the physical properties of the medium, Kirchoff's law must hold always, even if the medium is not in thermal equilibrium, and even in the medium is not a 


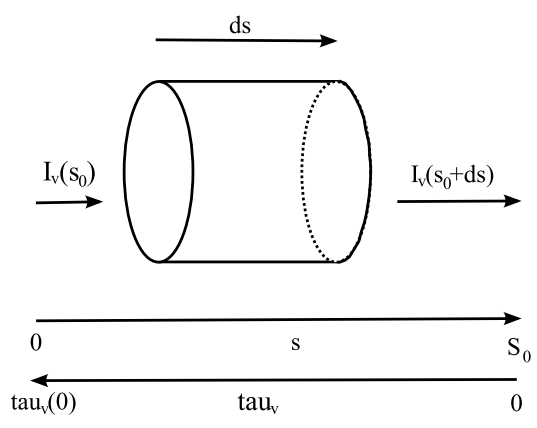

Figure 2: A sketch of the differential equation of the radiative transfer (see text).

perfect black-body. However, in these cases $B_{v}(T)$ is replaced by $S_{v}(T)$, and the Kirchoff's law is just a definition of what we call source function of the medium $S$. We can also express the "source function" as a function of the Plank law $S_{v}=B_{v}\left(T_{\mathrm{ex}}\right)$, but this is just a mere definition of the excitation temperature of the medium $T_{\mathrm{ex}}$, that of course can depend on the frequency: $S_{v}=B_{v}\left(T_{\text {ex }}(v)\right)$.

As we see, there is a tight relationship between the emitting and absorbing properties of all physical media. A perfectly transparent system can not emit radiation. On the contrary, if a medium emit radiation at a certain frequency, it can not possibly be fully transparent at this same frequency. Nevertheless, Kirchoff's law does not prohibit that a system could be transparent at some frequencies, being, at the same time, an emitter at other frequencies. This is in practice what happens in real media.

Let us define the opacity $\tau_{v}$ as a physical magnitude, whose total derivate, in the direction in which the ray is propagating, is precisely the absorption coefficient, but with the reversed sign: $-k_{v}$. Then:

$$
d \tau_{v}=-k_{v} d s \equiv \tau_{v}(s)=-\int_{\mathrm{s}}^{\mathrm{s}_{0}} k_{v} d s=\int_{\mathrm{s}_{0}}^{\mathrm{s}} k_{v} d s
$$

and the equation of radiative transfer can be rewritten in the following form:

$$
-\frac{1}{k_{v}} \frac{d I_{v}}{d s}=\frac{d I_{v}}{d \tau_{v}}=I_{v}-S_{v}
$$

where $S_{v}$ is the already mentioned "source function". Now we can solve the differential equation of the radiative transfer:

$$
I_{v}(s)=I_{v}(0) e^{-\tau_{v}(\mathrm{~s})}+\int_{0}^{\tau_{v}(\mathrm{~s})} S_{v} d \tau_{v}
$$

If $S_{v}$ is constant along the travelled path (and therefore independent of $\tau_{v}(s)$ and of $s$ ), we can integrate the equation, resulting in the widely used simplified version of the (integrated) equation of the radiative transfer:

$$
I_{v}(s)=I_{v}(0) e^{-\tau_{v}(\mathrm{~s})}+S_{v}\left(T_{\mathrm{ex}}(v)\right)\left(1-e^{-\tau_{v}(\mathrm{~s})}\right)
$$

In the end, the emergent radiation is the incident radiation, attenuated by the opacity of the medium by a factor $e^{-\tau_{v}(\mathrm{~s})}$, plus the source function $S_{v}\left(T_{\mathrm{ex}}(v)\right)$ of the medium multiplied by the factor one minus the opacity attenuation $\left(1-e^{-\tau_{v}(\mathrm{~s})}\right)$. 


\section{The emission spectrum of a grey-body}

According to the equation of transfer, for the black-body law to hold, we need to have a very large opacity at all frequencies and in all directions. Only in this case, and if the system is the thermodynamic equilibrium, we will have:

$$
\tau_{v}(s) \rightarrow \inf \quad \Longrightarrow \quad I_{v}(s)=S_{v}(T)=B_{v}(T)
$$

In practise, real systems are not perfectly opaque at all wavelengths, this is way we speak about the spectrum of emission of a grey-body.

As an example we will have a look at a semi-realistic case of a grey-body spectrum: a dusty envelope around a cool giant star. We will assume that the surface of the star is a perfect black-body characterized by a stellar temperature of $5600 \mathrm{~K}$, and that all the dust in the circumstellar cocoon is at a constant temperature $1800 \mathrm{~K}$. We will also assume that the radius of the dust cocoon is 10 times that of the star (100 times in surface). We will also neglect the contribution from the cosmic background at $2.7 \mathrm{~K}$.

The absorption coefficient of dust grains is not constant, but, in the Mie approximation, depends on the wavelength as follows (of course real circumstellar grains are much complex):

$$
k_{v}=0.5 \text { for } \lambda \leq \lambda_{0} \quad ; \quad k_{v}=0.5 \lambda_{0} / \lambda \text { for } \lambda \geq \lambda_{0}
$$

We will assume $\lambda_{0}$ equal to $0.5 \mu \mathrm{m}$. (The real value of this parameter depends on the size of the grains. In this case we are taking grains relatively small, with a diameter of about $1 \mu \mathrm{m}$.) The emergent spectrum will be

$$
I_{v}(s)=B_{v}(5600) e^{-\tau_{v}}+100 \times B_{v}(1800)\left(1-e^{-\tau_{v}}\right)
$$

Assuming a total opacity on the dust shell of 10 for $\lambda=\lambda_{0}$, we obtain the spectrum shown on figure (see Fig. 3). The dashed line shows the spectrum of the star in the absence of dust grains. In blue, we show the spectrum of a black-body at $1800 \mathrm{~K}$ and the same size as the dust cocoon (exactly what would happen if the opacity of the dust were large at all wavelengths).

\section{Equation of transfer with scattering}

To conclude this chapter, let us now take into account the effects of the scattering in the equation of transfer. We will now define $k_{v}^{\text {abs }}$ as the true absorption coefficient, and $k_{v}^{\text {sca }}$ as the scattering coefficient, which measures the probability of scattering per unit of travel length.

Normally, $k_{v}^{\text {sca }}$ can take different values depending on the angle $\Omega$ between the incident and scattered photon. We can define an average value of the scattering coefficient $\hat{k}_{v}^{\text {sca as: }}$

$$
\hat{k}_{v}^{\mathrm{sca}}=\frac{1}{4 \pi} \int_{0}^{4 \pi} k_{v}^{\mathrm{sca}}(\Omega) d \Omega
$$

With these definitions, the equation of transfer can we written in its differential form as follows: 


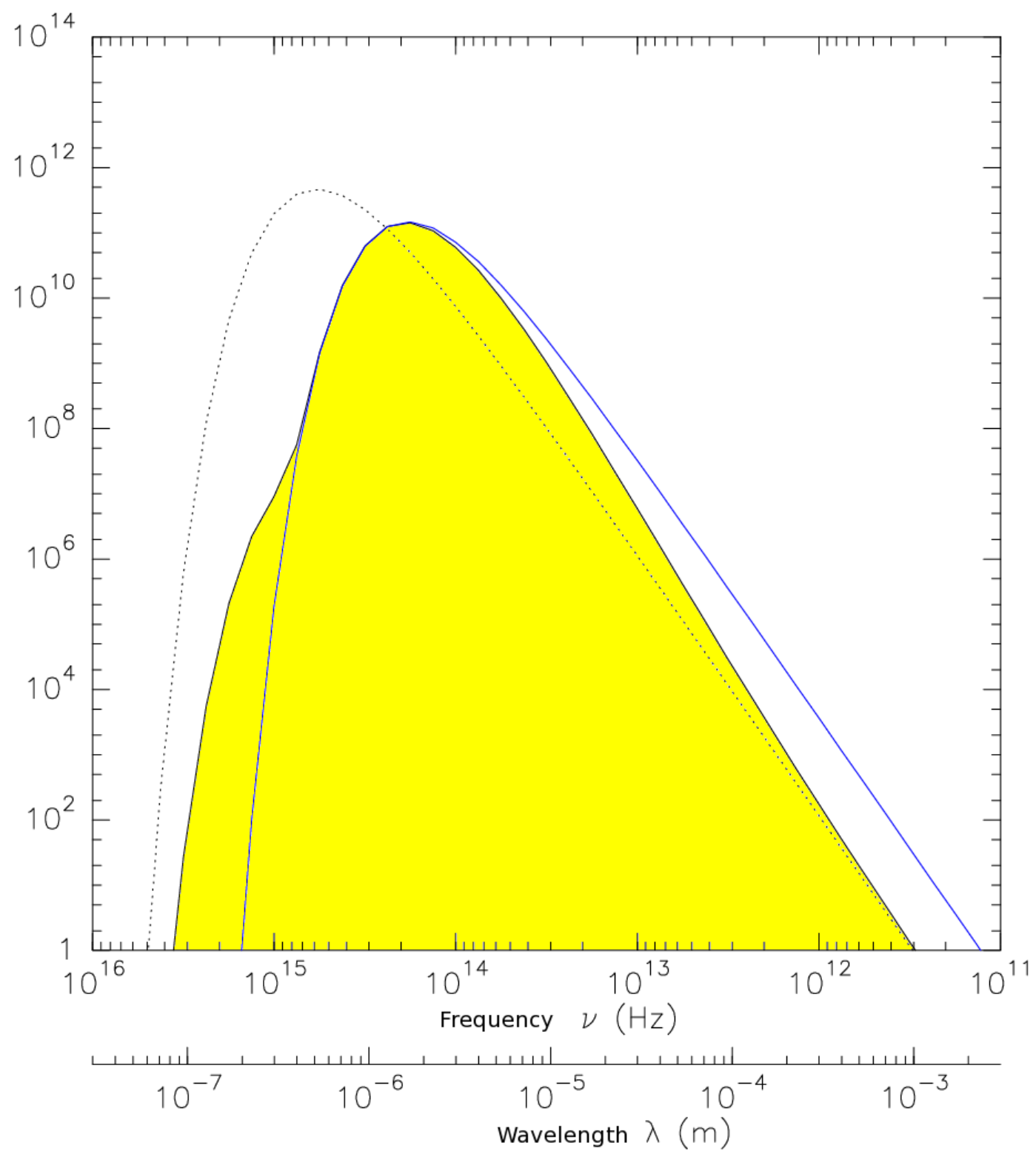

Figure 3: SEDs of a star surrounded by a dust cocoon as explained in the text (in yellow). The blue line shows the black-body spectrum of a system with the same temperature and size as the dust cocoon. The dashed line shows the spectrum of the star in absence of dust. 


$$
\frac{d I_{v}(\omega)}{d s}=-k_{v}^{\mathrm{abs}} I_{v}(\omega)-\hat{k}_{v}^{\mathrm{sca}} I_{v}(\omega)+\varepsilon_{v}+\frac{1}{4 \pi} \int_{0}^{4 \pi} k_{v}^{\mathrm{sca}}\left(\omega-\omega^{\prime}\right) I_{v}\left(\omega^{\prime}\right) d \omega^{\prime}
$$

where now $I_{v}\left(\omega^{\prime}\right)$ is the intensity of the radiation traveling in a different direction $\omega^{\prime}$. Usually a total extinction coefficient $k_{v}^{\text {ext }}$ is defined as $\hat{k}_{v}^{\text {sca }}+k_{v}^{\text {abs }}$. The albedo is simply the ratio between the pure scattering and total extinction, $\hat{k}_{v}^{\text {sca }} / k_{v}^{\text {ext }}$.

\section{References}

[1] J.D. Kraus, Radio Astronomy (2 ${ }^{\text {nd }}$ edition), Cygnus-Quasar Books.

[2] K. Rolfs, T.L. Wilson, Tools of Radio Astronomy ( $2^{\text {nd }}$ edition), Astronomy \& Astrophysics Library, Springer Verlag. 